\title{
Src blockade stabilizes a Flk/cadherin complex, reducing edema and tissue injury following myocardial infarction
}

\author{
Sara Weis,, ${ }^{1}$ Satoshi Shintani,, ${ }^{2}$ Alberto Weber, ${ }^{2}$ Rudolf Kirchmair, ${ }^{2}$ Malcolm Wood, ${ }^{1}$ \\ Adrianna Cravens, ${ }^{1}$ Heather McSharry, ${ }^{1}$ Atsushi Iwakura, ${ }^{2}$ Young-sup Yoon, ${ }^{2}$ Nathan Himes, ${ }^{3}$ \\ Deborah Burstein, ${ }^{3}$ John Doukas, ${ }^{4}$ Richard Soll, ${ }^{4}$ Douglas Losordo, ${ }^{2}$ and David Cheresh1
}

1'Department of Immunology, The Scripps Research Institute, La Jolla, California, USA. 'Division of Cardiovascular Research, St. Elizabeth's Medical Center, Tufts University School of Medicine, Boston, Massachusetts, USA. ${ }^{3}$ Department of Radiology, Beth Israel Deaconess Medical Center, Boston, Massachusetts, USA. ${ }^{4}$ TargeGen Inc., San Diego, California, USA.

\begin{abstract}
Ischemia resulting from myocardial infarction (MI) promotes VEGF expression, leading to vascular permeability (VP) and edema, a process that we show here contributes to tissue injury throughout the ventricle. This permeability/edema can be assessed noninvasively by MRI and can be observed at the ultrastructural level as gaps between adjacent endothelial cells. Many of these gaps contain activated platelets adhering to exposed basement membrane, reducing vessel patency. Following MI, genetic or pharmacological blockade of Src preserves endothelial cell barrier function, suppressing VP and infarct volume, providing long-term improvement in cardiac function, fibrosis, and survival. To our surprise, an intravascular injection of VEGF into healthy animals, but not those deficient in Src, induced similar endothelial gaps, VP, platelet plugs, and some myocyte damage. Mechanistically, we show that quiescent blood vessels contain a complex involving Flk, VE-cadherin, and $\beta$-catenin that is transiently disrupted by VEGF injection. Blockade of Src prevents disassociation of this complex with the same kinetics with which it prevents VEGF-mediated VP/edema. These findings define a molecular mechanism to account for the Src requirement in VEGF-mediated permeability and provide a basis for Src inhibition as a therapeutic option for patients with acute MI.
\end{abstract}

\section{Introduction}

Myocardial infarction (MI) leads to persistent post-ischemic vasogenic edema that develops as a result of increased vascular permeability (VP). Myocardial edema contributes to vessel collapse and impaired electrical function, including reperfusion arrhythmias and stunning, and could affect ventricular remodeling by changing myocardial stiffness (1). Therefore, reducing VP and the resulting edema is an attractive therapeutic approach for the treatment of acute MI. VEGF, first described as "vascular permeability factor" (2), likely contributes to myocardial edema, as it is expressed within hours following ischemic injury and potently induces VP. Accordingly, while VEGF can lead to long-term angiogenesis and vessel collateralization, it is possible that the VP-promoting effects of VEGF early in this disease can contribute to some of the pathology associated with ischemic injury. Thus, it might be highly advantageous to disrupt the early VPpromoting activity of VEGF without affecting its angiogenic activity.

Recently, we reported that mice deficient in pp60Src showed no VP response to VEGF and displayed minimal edema and infarction volume following stroke (3). Importantly, these mice showed a normal angiogenic response to VEGF (4), suggesting that Src kinase

Nonstandard abbreviations used: endothelial cell (EC); left anterior descending (LAD); left ventricle (LV); myocardial infarction (MI); Src family kinase (SFK); vascular permeability (VP).

Conflict of interest: R. Soll and J. Doukas are employed by TargeGen Inc. and together with D. Cheresh have stock in this company. D. Cheresh is on the Scientific Advisory Board of TargeGen but is not an employee, board member, or a recipient of research funding from the company. TargeGen is developing small molecule therapies for use in treating ischemic diseases; however, these molecules are independent from those described in the current manuscript.

Citation for this article: J. Clin. Invest. 113:885-894 (2004). doi:10.1172/JCI200420702. may play a specific role in the VEGF physiological response by regulating VP. In normal mice, pharmacological blockade of Src kinases similarly reduced edema and infarction volume following stroke. These findings suggested it might be possible to control ischemic injuries by regulating VEGF-mediated Src activity. Here, we present ultrastructural and biochemical evidence to explain how VEGFmediated Src kinase activity in blood vessels regulates endothelial cell (EC) barrier function following MI.

Previous studies have shown that EC barrier function depends in part on VE-cadherin, an endothelial-specific cadherin (5). Recent evidence suggests that Src kinases play a general role in regulating cadherin function on a wide variety of cell types $(6,7)$. In fact, Src kinase can phosphorylate E-cadherin, causing epithelial cells to dissociate from one another (6). These findings and the fact that Src is recruited to the VEGF receptor Flk upon VEGF binding (8) prompted us to consider whether EC barrier function could be disrupted by VEGFmediated Src regulation of VE-cadherin function. In this study, we isolated a preformed complex between Flk, VE-cadherin, and $\beta$-catenin from normal quiescent blood vessels. Upon VEGF stimulation of these blood vessels in vivo, this Flk/cadherin complex transiently dissociated. Importantly, blockade of Src kinase prevented the dissociation of this complex, making blood vessels resistant to VEGF-mediated VP. These findings were supported by ultrastructural studies in which Src blockade led to the elimination of VEGF-induced EC gaps. To our surprise, these gaps were often plugged with activated platelets that appeared to reduce vessel patency in the area of the ischemic injury, thereby contributing to the reduction in blood flow to this region. These adherent/activated platelets, which likely contribute to the VEGF quantity within this microenvironment (9), may enhance the VP response in these tissues. Thus, by blocking Src following an 
ischemic injury, it is possible to disrupt a VEGF-mediated physiological cascade that contributes to the severity and longevity of an ischemic lesion such as MI.

\section{Methods}

Src family kinase (SFK) inhibitors. PP1, developed by Pfizer (now available from BIOMOL Research Laboratories Inc., Plymouth Meeting, Pennsylvania, USA), inhibits enzymatic activity of Lck, Lyn, and Src at $\mathrm{IC}_{50}$ values of 5, 6, and $170 \mathrm{nM}$, respectively (10). PP1 was used at $0.5-3 \mathrm{mg} / \mathrm{kg}$, equivalent to $22-133 \mathrm{nM}$ for a mouse blood volume of $2 \mathrm{ml}$. SKI-606, developed by Wyeth-Ayerst Research (Pearl River, New York, USA) $(11,12)$, inhibits Src at $1.2 \mathrm{nM}(12)$. SKI-606 was used at $0.5-5 \mathrm{mg} / \mathrm{kg}$, equivalent to $12-118 \mathrm{nM}$ in the mouse.

Ischemic models. For analysis of infarct size and myocardial water content, MRI studies, and echocardiographic function and fibrotic tissue experiments, we used a rat model of acute MI with permanent occlusion of the left anterior descending (LAD) coronary artery, as described (13). A similar mouse model of MI was used to assess the effect of Src blockade on infarct size, edema, and tissue ultrastructure after permanent LAD occlusion. Adult male mice 8-12 weeks old were used for all studies, except 2-year-old C57BL/6 mice were used as a model of severe MI to test the effects of Src inhibition on survival. The effect of Src inhibition on infarct size during transient ischemia was tested using a rat ischemia-reperfusion model with temporary LAD occlusion for 60 minutes (SKI-606) or 45 minutes (PP1), administration of test agent 60 minutes later, and determination of infarct size 24 hours later. Adult male Sprague-Dawley rats (Harlan, Indianapolis, Indiana, USA) and C57BL/6, $\mathrm{pp}^{\mathrm{C}} 6 \mathrm{Src}^{-/-}$, and $\mathrm{pp} 60 \mathrm{Src}^{+-}$mice (14) (Jackson Laboratory, Bar Harbor, Maine, USA) were maintained and used under approved Animal Subjects protocols at The Scripps Research Institute, Tufts University, or TargeGen Inc.

Infarct size. After 24 hours, 10\% Evans blue (Sigma-Aldrich, St. Louis, Missouri, USA) was injected intravenously before sacrifice. Hearts were removed and cut in three equivalent sections distal to the occluding LAD suture and one proximal to it. Images of the distal sections were digitized using NIH Image software to evaluate the nonperfused area at risk. Sections were stained with $2 \%$ triphenyltetrazolium chloride (Sigma-Aldrich) to delineate ischemic area. This method correlates well with histological measurements (15). Infarct size is presented as the percentage of area at risk to eliminate variability.

Water content and cardiac function. Previous studies have used MRI to assess myocardial edema (16). In this study, in vivo water content was evaluated using MRI performed serially on anesthetized rats 24 hours following MI using a 4.7-T MR scanner (Bruker, Billerica, Massachusetts, USA). Adult male rats were administered PP1 (5.0 mg/kg intraperitoneally), SKI-606 (5.0 mg/ kg i.v.), or vehicle 45 minutes following permanent LAD occlusion. MRI experiments to quantify $\mathrm{T} 2$ values of the myocardium were conducted by applying an ECG and respiratory-triggered multiecho spin echo sequence (number of echoes, 8; echo time, 6.6 milliseconds; slice thickness, $1.0 \mathrm{~mm}$; inplane resolution, $430 \mu \mathrm{m}^{2}$; total slices, six to seven). The trigger delay was chosen to capture all echoes during full diastole to avoid motion artifact between echoes. Previous studies have determined the T2 values of normally perfused myocardium ( $27 \pm 6.3$ milliseconds; N. Himes, data not shown). Corresponding gradient echo images were acquired for each slice to clearly delineate the blood/myocardium border for region-ofinterest evaluation of the spin echo sequence. Regions with T2 values greater than 40 milliseconds (two standard deviations above the mean of normally perfused myocardium) were delineated and the volume was calculated as a percentage of the total LV myocardial volume. In addition, the ex vivo myocardial water content of proximal heart sections was measured as the percentage difference between initial wet and dry weights after 24 hours of incubation at $80^{\circ} \mathrm{C}$. Transthoracic echocardiography (SONOS 5500; Agilent Technologies, Palo Alto, California, USA) was performed to evaluate LV function before (baseline) and 4 weeks after MI. For this analysis, rats were anesthetized with $0.6 \mathrm{ml} / \mathrm{kg}$ ketamine intraperitoneally. The regional wall motion score was calculated as described previously (17).

Fibrotic tissue. For the histopathological analysis of fibrotic tissue, hearts were removed after functional analysis, and the volume and circumference of fibrotic tissue was determined by staining with elastic trichrome and computer-based planimetry. The amount of fibrotic tissue was measured as the percentage of LV area as well as the percentage of LV circumference to eliminate the contribution of differences in end-diastolic diameter and hypertrophy among the groups.

In vivo permeability model. Adult mice $8-12$ weeks old were injected i.v. with $50 \mu \mathrm{l}$ of the Src inhibitor PP1 $(1.5 \mathrm{mg} / \mathrm{kg}$ in PBS/DMSO; BIOMOL Research Laboratories) 5 minutes prior to being injected with $100 \mu \mathrm{l}$ of VEGF or bFGF $(0.2 \mathrm{mg} / \mathrm{kg}$ in PBS; PeproTech, Rocky Hill, New Jersey, USA). At the appropriate time, the heart was rapidly excised and homogenized in $3 \mathrm{ml}$ RIPA lysis buffer as previously described (4) and the protein concentration was measured (BCA Protein Assay; Pierce, Rockford, Illinois, USA).

Ultrastructural analysis by electron microscopy. Cardiac tissue was prepared from 8- to 12-week-old mice following VEGF injection or 3-24 hours following ischemia, and the infarct, the peri-infarct, and remote regions were sectioned. Tissue was fixed in $0.1 \mathrm{M}$ sodium cacodylate buffer ( $\mathrm{pH} 7.3$ ) containing $4 \%$ paraformaldehyde and $1.5 \%$ glutaraldehyde for 2 hours, transferred to $5 \%$ glutaraldehyde overnight, then to $1 \%$ osmium tetroxide for 1 hour. Blocks were washed, dehydrated in a graded ethanol series, and embedded in Epon/Araldite resin. Ultrathin sections were stained with uranyl acetate and lead citrate and were viewed using a Philips CM-100 transmission electron microscope.

Immunoprecipitation and immunoblotting. Tissue lysates were prepared for immunoprecipitation and immunoblotting as previously described (4) with antibodies from Santa Cruz Biotechnology (SC; Santa Cruz, California, USA) or Biosource International (B; Camarillo, California, USA): Flk (SC315), VE-cadherin (SC6458), $\beta$-catenin (SC7963), phospho-tyrosine (SC7020 or SC508), Src phospho-Y418 (B44-660), and FAK phospho-Y861 (B44-626). Representative data from at least three separate experiments are shown.

Statistical analysis. Data are presented as mean \pm SEM, with statistical significance determined from Student's $t$-test $(P<0.05)$.

\section{Results}

\section{Functional effects of Src inbibition following MI}

Src blockade reduces edema and provides protection following MI. To establish the potential role of Src in the pathophysiology following MI, we investigated the effects of Src deletion on the murine heart following ligation of the LAD coronary artery. Twenty-four hours after the onset of ischemia, adult male 8- to 12-week-old $\mathrm{pp} 60 \mathrm{Src}^{-1-}$ mice had significantly decreased myocardial water content $(P<0.01)$ associated with $50 \%$ smaller infarct size $(P<0.001)$ compared with heterozygous controls ( $n=4$ for each group; Figure $1 \mathrm{~A})$. We have 
A
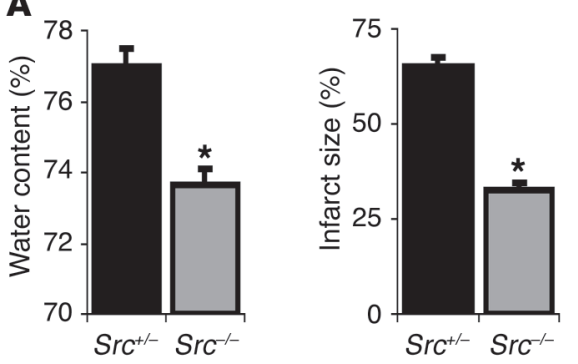

C

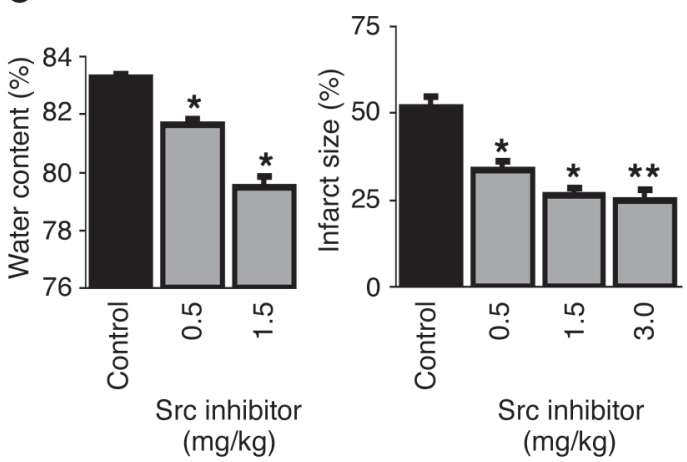

B
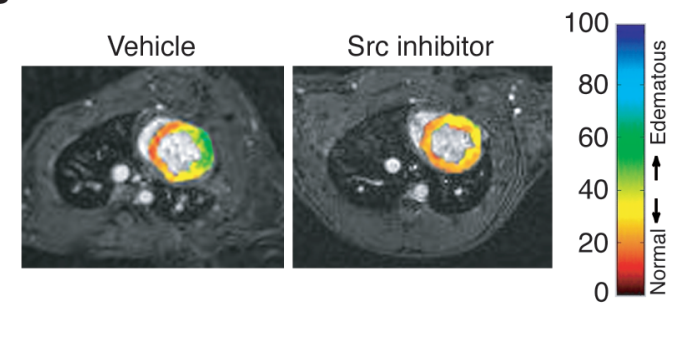

D

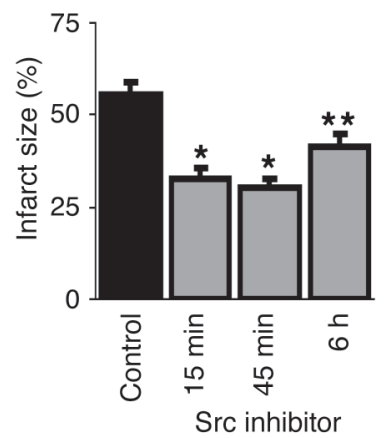

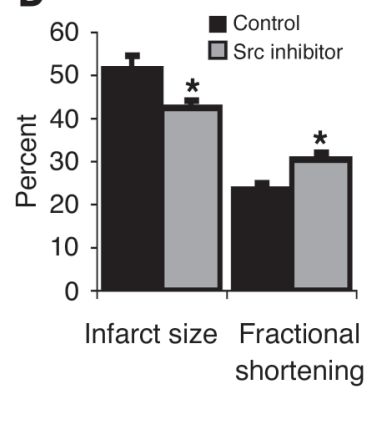
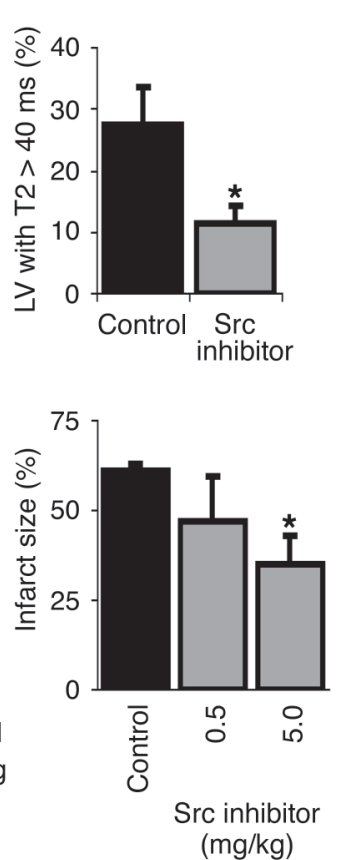

Figure 1

Src blockade protects following myocardial infarction. (A) pp60Src ${ }^{-1-}$ mice have significantly reduced myocardial water content and infarct size 24 hours after MI. (B) MRI T2 maps overlaid on gradient echo images in rats treated with vehicle or the PP1 Src inhibitor. Scale at right indicates T2 values from red (lower T2) to blue (higher T2). T2 values greater than 40 milliseconds were used as an index of edema, and representative images reveal reduced volume containing T2 values greater than 40 milliseconds 24 hours following MI in PP1-treated rats. Graph shows significant differences of the percentage of LVs with T2 values greater than 40 milliseconds between vehicle- and SKI-606 treated rats. (C) Treatment with a Src inhibitor results in significant and dose-dependent decreases in myocardial water content and infarct size after MI. Single-dose treatment with a Src inhibitor was optimally effective in reducing infarct size when administered 45 minutes after LAD ligation and still reduced infarct size significantly when administered up to 6 hours after infarct. (D) Src inhibition reduces infarct size and preserves function following transient ischemia and reperfusion. All panels represent the Src inhibitor PP1, except for B, as noted, and D, right panel, in which the Src inhibitor SKI-606 was used. ${ }^{*} P<0.05 ;{ }^{* *} P<0.001$.

previously reported that the $p$ p60Src ${ }^{+/-}$mice show a normal permeability and signaling response to VEGF (4), and these mice are genetically most similar to the $p$ p $60 \mathrm{Src}^{-1-}$ mice, which allows us to establish a strong relationship between Src expression, VP, and infarct volume following MI. VEGF expression following MI was similar between genotypes (data not shown), demonstrating Src inhibition did not interfere with induction of VEGF, but rather influenced a downstream effector.

MRI has previously been utilized to assess myocardial viability (18) and edema (16). In this study, to detect the spatial distribution of edematous myocardium we used MRI to evaluate short axis maps of the parameter T2 of the left ventricle (LV) obtained 24 hours following permanent LAD coronary artery occlusion in adult male rats receiving PP1 $(n=2)$, SKI-606 $(n=5)$, or vehicle $(n=5)$. Because of their increased water content, edematous regions are expected to have a longer $\mathrm{T} 2$ relaxation than nonedematous regions. Therefore, regions with T2 values greater more than 40 milliseconds (two standard deviations above normally perfused myocardium) were delineated as an index of edema and expressed as percentage of total LV volume. Compared with vehicle, PP1 treatment reduced the extent of edema, shown in Figure $1 \mathrm{~B}$ as green regions with T2 values greater than 40 milliseconds. Similarly, the index of edema as defined was 59\% less in SKI-606-treated rats than in vehicle-treated rats $(P<0.05$; Figure 1B, graph). To validate these findings, myocardial water content was also computed ex vivo using wet/dry weights of nonischemic myocardium (Figure 1C). PP1 provided dose-dependent decreases in edema and infarct size, with a maximum decrease at $1.5 \mathrm{mg} / \mathrm{kg}(n>5$ for each group; $P<0.001$; Figure 1C). PP1 also produced a significant reduction in infarct size when administered following permanent occlusion in the mouse and rat (data not shown). This effect was time dependent with maximum benefit ( $50 \%$ smaller infarct size) achieved with treatment 45 minutes following occlusion, yet treatment after 6 hours still yielded $25 \%$ protection $(n=5$ for each group; $P<0.05$; Figure 1C).

Src blockade provides protection following transient ischemia. To establish whether Src inhibition is beneficial following transient ischemia and reperfusion, adult male rats were subjected to occlusion followed by reperfusion and then were evaluated for ventricular function and infarct size after 24 hours. Src inhibition by PP1 preserved LV fractional shortening and reduced infarct size compared with controls ( $n=4$ for each group; $P<0.05$; Figure 1D). The $18 \%$ reduction in infarct size following ischemia-reperfusion compares to a $50 \%$ decrease following permanent occlusion in which the hypoxic stimulus driving VEGF expression is maintained. In addition, SKI-606 $(5 \mathrm{mg} / \mathrm{kg})$ provided a $43 \%$ decrease in infarct size in the ischemia-reperfusion model $(n=5$ for each group; $P<0.01$; Figure 1D). Collectively, these data support the possibility of a 

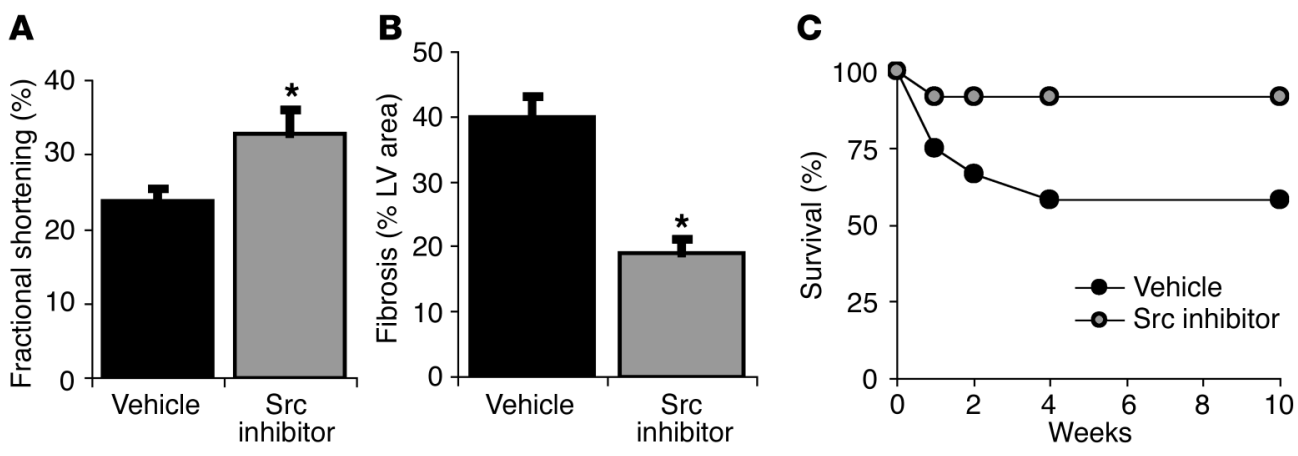

Figure 2

Effects of Src blockade on the long-term consequences of MI. Src inhibition delivered once 45 minutes after occlusion provides long-term protection even after 4-10 weeks. (A) Echocardiographic assessment reveals left ventricular function (\% Fractional shortening) is improved after 4 weeks in rats that received the Src inhibitor. (B) Histological examination reveals less fibrosis (\% LV area) at 4 weeks after occlusion in rats treated with the Src inhibitor. (C) Src inhibition improves survival of 2-year-old C57 black mice, a model that typically shows more than $40 \%$ mortality by 10 weeks. ${ }^{*} P<0.05$.

beneficial effect of Src inhibition following transient ischemia as well as permanent occlusion.

\section{Effects of Src blockade on the long-term consequences of MI including cardiac function, fibrosis, and survival}

Ventricular function (4 weeks). To monitor the long-term effect of a single injection of a Src inhibitor on left ventricular function, echocardiography was performed either 24 hours or 4 weeks following MI on animals treated with the Src inhibitor or vehicle control. Echocardiography revealed Src inhibition immediately following MI offered $46 \%$ preservation of fractional shortening (Figure $2 \mathrm{~A} ; n=8$ for each group; $P<0.05)$ and diastolic LV diameter $(11 \% ; n=8 ; P<0.05)$ over 4 weeks compared with animals receiving vehicle control, indicating that contractile function in the rescued tissue was preserved in the long term. Src inhibition also provided a favorable effect on systolic LV diameter $(16 \% ; n=8 ; P<0.05)$ and regional wall motion $(9 \% ; n=8$; $P<0.05)$. Treatment with the SKI-606 Src inhibitor also favorably affected fractional shortening and regional wall motion score after 24 hours $(n=7$ for each group; $P<0.01)$.

Fibrosis (4 weeks). Another long-term consequence following MI is the accumulation of chronic myocardial fibrotic tissue, a direct reflection of the extent of tissue necrosis following MI. To evaluate the effect of initial Src inhibition on fibrosis 4 weeks after MI in rats, histopathological analysis of fibrotic tissue was performed using elastic trichrome staining. A single injection of a Src inhibitor delivered 45 minutes following injury contributed to a $52 \%$ decrease in LV fibrotic tissue compared with control (Figure 2C; $n=4$ each group; $P<0.01$ ), and better preservation of myocardial fibers and LV architecture, as measured 4 weeks following injury.

Survival (10 weeks). To monitor the effect of Src inhibition on survival, we used 2-year-old C57 black mice, as occlusion of the LAD coronary artery in these animals typically results in approximately $40 \%$ mortality 10 weeks following MI (19). This procedure led to the expected mortality rate in control animals; however, administration of PP1 $(1.5 \mathrm{mg} / \mathrm{kg}) 45$ minutes after MI increased survival compared with that of controls 10 weeks following injury (Figure 2B; $91.7 \%$ vs. $58.3 \%$ survival, respectively; $n=12$ for each group). Together, these findings suggest that blocking Src shortly after MI has both shortterm and long-term benefits associated with limiting cardiac damage and fibrosis, thereby increasing survival.
Ultrastructural effects of Src inbibition following MI Effect of MI on vascular integrity and myocyte viability in the peri-infarct zone. To characterize the mechanism associated with the VEGFmediated VP response, we evaluated cardiac tissues at the ultrastructural level following MI. We investigated the mechanism of permeability by assessing the ultrastructural effects of Src inhibition on small vessels in this region 3-24 hours after $\mathrm{MI}$ in adult male mice 8-12 weeks old. A summary of observations for 250 blood vessels examined per group using transmission electron microscopy is provided in Table 1. In contrast to normal myocardial tissue (Figure 3, A-C), we found numerous examples of impaired vascular barrier function and/or cellular damage in the peri-infarct zone at both 3 and 24 hours following MI. At 3 hours, extravasated blood cells (rbc's, platelets, and neutrophils) were present in the interstitium, apparently having escaped from nearby vessels (Figures 3D and 4). However, at 24 hours, some ECs were swollen and occluded part of the vessel lumen (Figure 3, E and F), often appearing electron lucent and containing many caveolae. Large round vacuoles were present in the endothelium, often several times larger than the EC thickness (Figure 3G). Myocyte injury increased with time following MI and varied between adjacent cells, and was identifiable as mitochondrial rupture, disordered mitochondrial cristae, intracellular edema, and myofilament disintegration (Figures $3 \mathrm{H}$ and 4). The myocytes most affected were often adjacent to injured blood vessels or free blood cells (Figure 4). We frequently observed neutrophils (Figure 3I) 24 hours after MI, which participate in the acute response to injury and may contribute to VEGF production (20).

\section{Table 1}

Ultrastructural observations in mouse cardiac tissue following MI or VEGF injection

\begin{tabular}{lcccc}
\hline & $\begin{array}{c}\text { EC barrier } \\
\text { dysfunction } \\
\text { and adhesion }\end{array}$ & $\begin{array}{c}\text { Platelet } \\
\text { activation }\end{array}$ & $\begin{array}{c}\text { EC } \\
\text { injury }\end{array}$ & $\begin{array}{c}\text { Cardiac } \\
\text { damage }\end{array}$ \\
$3 \mathrm{~h}$ after Ml & 18 & 36 & 31 & 22 \\
$3 \mathrm{~h}$ after Ml + PP1 & 2 & 11 & 14 & 2 \\
$24 \mathrm{~h}$ after Ml & 5 & 7 & 34 & 45 \\
$24 \mathrm{~h}$ after Ml + PP1 & 0 & 1 & 15 & 9 \\
Control & 0 & 0 & 1 & 0 \\
VEGF, pp60Srct+ & 24 & 18 & 33 & 16 \\
VEGF, pp60Sr- & 0 & 0 & 0 & 0 \\
\hline
\end{tabular}

For each group, murine left ventricular tissue was examined for 4 hours (approximately 250 microvessels) on a transmission electron microscope, and observations were counted and grouped according to EC barrier dysfunction (gaps, fenestrations, extravasated blood cells), platelet activation/adhesion (platelets, degranulated platelets, platelet clusters, platelet adhesion to ECM), EC injury (electron-lucent ECs, swollen ECs, large EC vacuoles, occluded vessel lumen), and cardiac damage (mitochondrial swelling, disordered cristae, myofilament disintegration). 

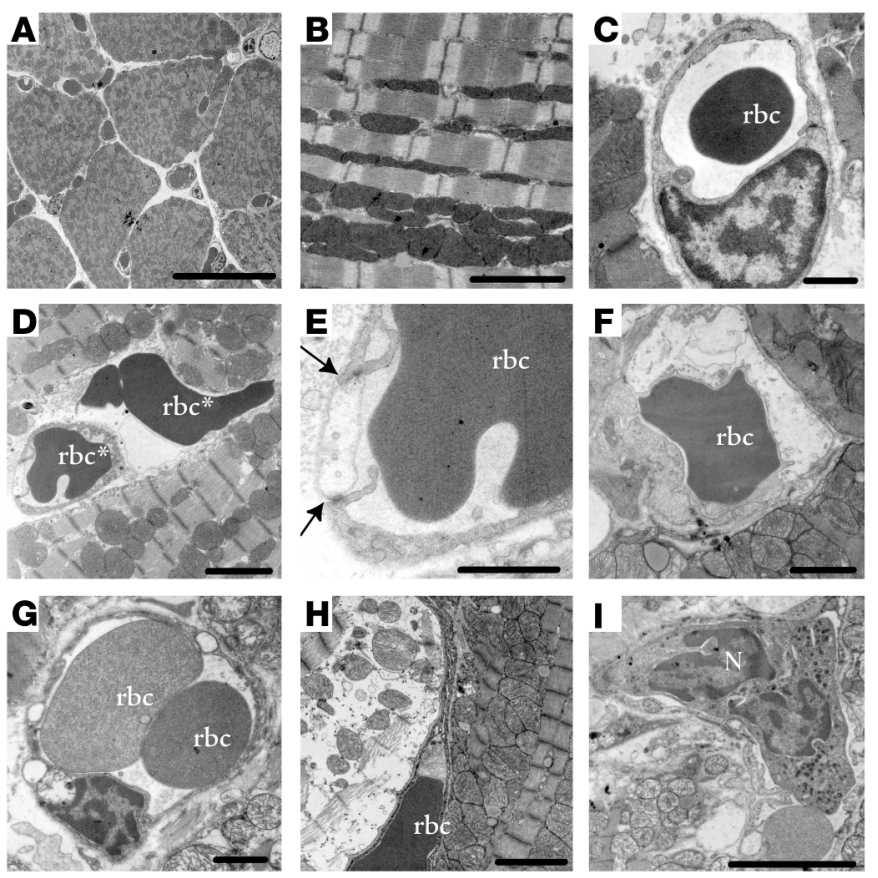

\section{Figure 3}

Ultrastructural changes to cardiac microvessels following MI or VEGF injection in mice. (A) Normal ventricular myocardium observed in a lowpower transmission electron micrograph cross-section of cardiac myocytes and blood vessels. Scale bar: $20 \mu \mathrm{m}$. (B) Section transverse to myocytes showing normal myofilament architecture and mitochondria. Scale bar: $2 \mu \mathrm{m}$. (C) An rbc in the lumen of a normal microvessel with intact interendothelial junctions and consistent thickness of the endothelial layer. Scale bar: $1 \mu \mathrm{m}$. (D-I) Ultrastructural damage to blood vessels and ventricular myocardium from the peri-infarct region following $\mathrm{MI}$ or from left ventricular tissue following systemic VEGF injection. Images are representative, taken from either group. Summary of results appears in Table 1. (D) An rbc in the extracellular space adjacent to an abnormal blood vessel. Scale bar: $2 \mu \mathrm{m}$. "rbc" indicates red blood cell inside blood vessel; "rbc" indicates red blood cell in extracellular space. (E) Enlargement of blood vessel in $\mathbf{D}$, showing impaired interaction (arrows) between a swollen, electron-lucent EC and a neighboring EC. Scale bar: $1 \mu \mathrm{m}$. $(\mathbf{F})$ Swollen, electron-lucent EC appears to restrict passage of rbc's through vessel lumen. Scale bar: $1 \mu \mathrm{m}$. (G) Vessel with no apparent gaps, but three large vacuoles apparent in endothelium. Scale bar: $1 \mu \mathrm{m} .(\mathbf{H})$ Severely affected myocyte (left) in peri-infarct zone with disintegrating myofilaments and mitochondria. Adjacent myocyte (right) appears less damaged. Scale bar: $2 \mu \mathrm{m}$. (I) Neutrophil (N) in blood vessel near myocyte damage. Scale bar: $5 \mu \mathrm{m}$.

Accumulation of microthrombi in EC gaps during the early response following MI. Within 3 hours following MI, the first noticeable ultrastructural changes detected in blood vessels were gaps between ECs with exposed basement membrane or ECM. To our surprise, many of these gaps were plugged by microthrombi containing activated/adherent platelets (Figure 5). Platelets were observed in direct contact with the basal lamina exposed between ECs (Figure 5, A-D) or with the underlying stroma (Figure 5, E and F). Many of the platelets at these sites were degranulated (Figure 5, C and D), suggesting the platelets were activated and their contents (possibly including VEGF) had been deposited within the microenvironment.

In a number of circumstances, these platelets appeared to restrict blood flow. In Figure 6, three microvessels are shown in which platelet aggregates impair vessel patency to varying extents. Seven platelets, two with narrow protrusions through the discontinuous endothelium, do not impede blood flow through a larger microvessel (Figure 6, A and B). Most of the vessel lumen is occluded in the vessel shown in Figure 6, C and D, in which an aggregate of ten mostly degranulated platelets limits blood flow to one rbc. In contrast, Figure 6, E and F, shows a vessel with a platelet aggregate dominating the entire vessel lumen.

Early Src blockade prevents VP and myocyte damage at 24 hours following injury. To test whether Src inhibition could block microvascular hyperpermeability at the ultrastructural level, we treated animals with PP1 $(1.5 \mathrm{mg} / \mathrm{kg})$ or vehicle 45 minutes following coronary artery occlusion. Src inhibition dramatically protected the periinfarct region from endothelial barrier dysfunction and blood vessel damage (Table 1). The most notable result was the effect of PP1 at 24 hours, revealing a significant reduction in myocyte injury. While PP1 did not abrogate all evidence of damage, it did prevent vascular gaps, resulting in a vastly improved EC ultrastructural appearance and providing protection to the blood vessels and myocytes. These results provide an ultrastructural basis for the improvement in ventricular function and survival measured at 24 hours after MI in the animals receiving a Src inhibitor.

\section{MI and systemic VEGF injection produce a comparable ultrastructural response}

To further reveal the mechanism of permeability and determine the contribution of VEGF to this pathology, we injected $0.2 \mathrm{mg} / \mathrm{kg}$ VEGF i.v. into normal adult male mice and evaluated cardiac tissue at the ultrastructural level. To our surprise, the extent of endothelial barrier dysfunction, platelet adhesion, and vessel injury at 30 minutes following VEGF administration was comparable to that seen in the peri-infarct zone after MI 3 hours following injury

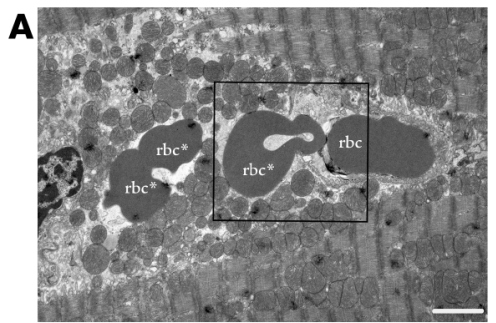

B

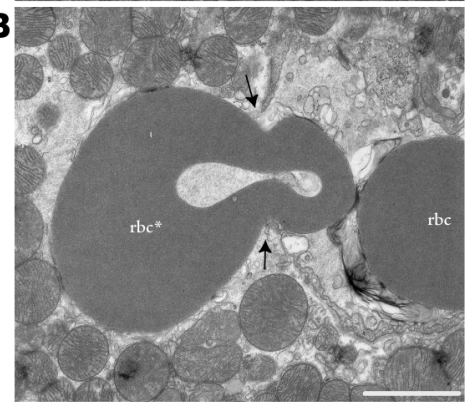

Figure 4

Vascular leak at the ultrastructural level. (A) Microvessel containing one $\mathrm{rbc}$ and another rbc ( $\mathrm{rbc}^{\star}$ ) that is in the process of extravasation (boxed). Two other extravasated rbc's $\left(\mathrm{rbc}^{*}\right)$ are to the left. Note severe edema and disruption of myofilaments. Scale bar: $2 \mu \mathrm{m}$. (B) Enlargement of the boxed region in A showing extravasation site. Arrows indicate gap between ECs. Scale bar: $2 \mu \mathrm{m}$. 

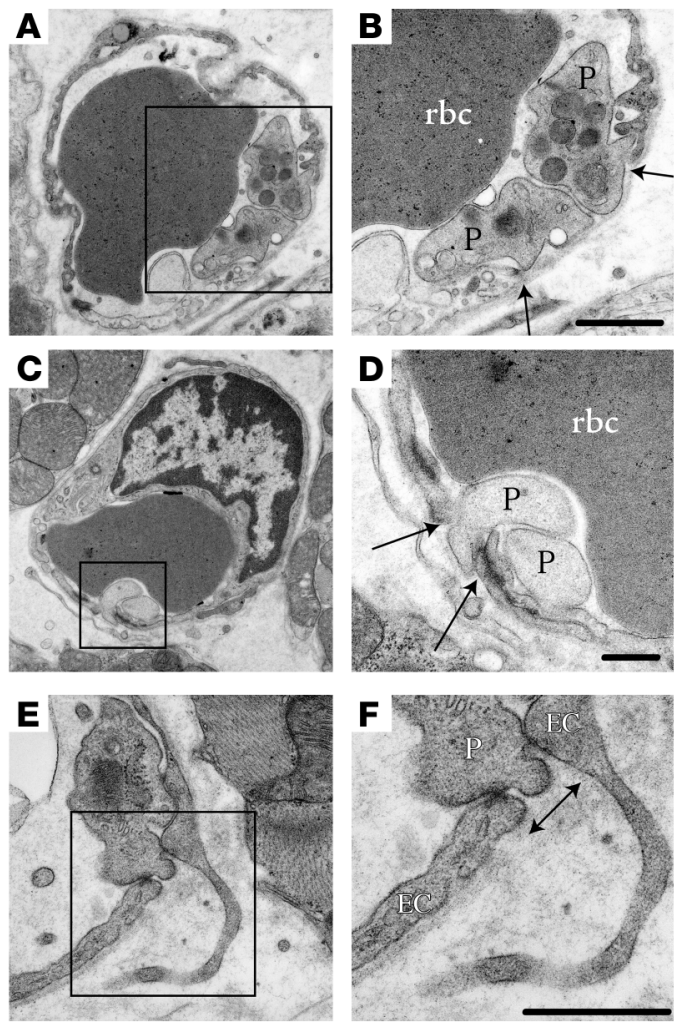

(Table 1). We found similar evidence of damage in the brain following systemic VEGF injection (data not shown), indicating these effects are likely to be systemic. These results suggest VEGF-mediated VP parallels many of the vascular effects following MI. The fact that Src-deficient mice were protected following MI and lacked VP in the skin and brain following local VEGF injection (4) prompted us to determine whether these animals were spared VEGF-induced VP in the heart. Consistent with the Src inhibitor results, no signs of vascular gaps or injury response following VEGF injection were seen in the pp60Src-1- animal (Table 1).

\section{Biochemical mechanism to account for the role of Src in EC barrier function}

Flk-cadherin-catenin complex maintains endothelial barrier function. To more fully understand the mechanism underlying Src regulation of VEGF-mediated VP, we examined structural elements that contribute to endothelial junctional integrity. Previous in vitro studies have implicated growth factors in the regulation of cadherin function (21-24). In cultured ECs under flow conditions, VE-cadherin forms a complex with Flk (25), which may contribute to cell-cell

\section{Figure 6}

Platelet aggregates impair blood flow through occluded vessels. (A-F) Microvessels containing rbc's and platelet aggregates that protrude narrow extensions through ( $\mathbf{A}$ and $\mathbf{B}$ ) or interact with $(\mathbf{C}-\mathbf{F})$ discontinuous endothelium (arrows). $\mathrm{P}^{\star}$, platelets appearing to directly contact basement membrane. Some platelets appear to be degranulated and/or have a less rounded shape, consistent with platelet activation. The platelet aggregates appear as five to eight platelets in plane of section and impede flow through part ( $\mathbf{A}$ and $\mathbf{B})$, most (C and $\mathbf{D})$, or all ( $\mathbf{E}$ and $\mathbf{F})$ of the vessel lumen. Scale bars: $1 \mu \mathrm{m}$ (B) and $500 \mathrm{~nm}(\mathbf{D}-\mathbf{F})$.

\section{Figure 5}

Platelet adhesion in blood vessels following MI or VEGF injection. (A and B) Microvessel containing an rbc with gap (arrows) between adjacent ECs (one of which appears electron lucent) in which two platelets $(P)$ interact with intact basal lamina. Scale bar: $500 \mathrm{~nm}$. (C and D) Gap in endothelial barrier (arrows) plugged by two degranulated platelets. Scale bar: $500 \mathrm{~nm}$. (E and F) Platelet appears to interact with ECs to plug gap (arrow). Boxed regions in $\mathbf{A}, \mathbf{C}$, and $\mathbf{E}$ correspond with enlargements in B, D, and F, respectively. Scale bar: $500 \mathrm{~nm}$.

junctional integrity. Therefore, we considered if such a complex could be detected in vivo and whether it was subject to dissociation by VEGF. Heart lysates prepared from adult mice injected with VEGF or with vehicle only were subjected to immunoprecipitation with anti-Flk followed by immunoblotting for VE-cadherin and $\beta$-catenin. In control mice, we isolated a pre-existing complex between Flk, $\beta$-catenin, and VE-cadherin in blood vessels. This complex was rapidly disrupted within $2-5$ minutes following systemic VEGF stimulation and had reassembled by 15 minutes in blood vessels in vivo (Figure 7, A and B). In control experiments, these events were not observed following injection of bFGF (Figure 7A), an angiogenic growth factor that does not promote VP. The time scale of complex dissociation by VEGF paralleled that of Flk, $\beta$-catenin, and VE-cadherin phosphorylation and the dissociation of $\beta$-catenin from VE-cadherin (Figure 7C). These VEGF-mediated events were Src dependent, as the Flk-cadherin-catenin signaling complex remained intact and phosphorylation of $\beta$-catenin and VE-cadherin did not occur in VEGF-stimulated mice treated with Src inhibitors (Figure 7D). Src inhibitor treatment blocks VEGF-induced Src activity in a dose-dependent way in vivo as assessed with antibodies for Src phospho-Y418 and the Src substrate FAK-phospho-Y861 (Figure 7E). This biochemical profile strongly correlates with our
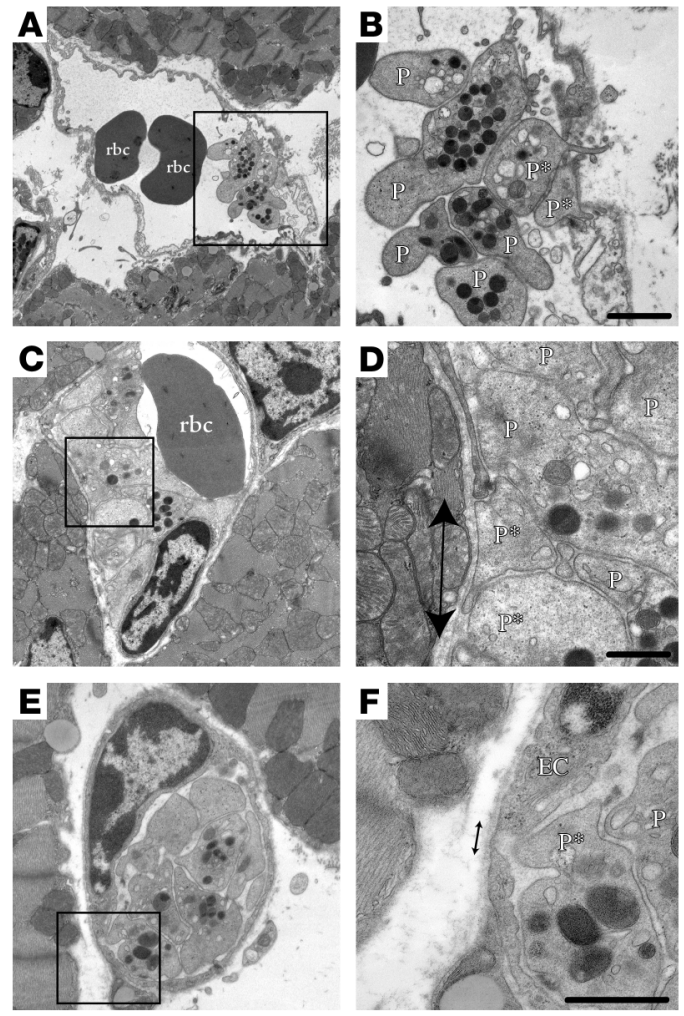
A

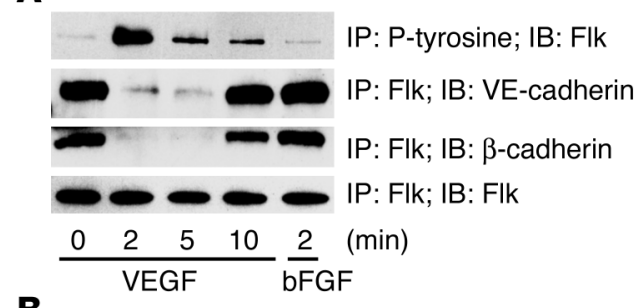

B

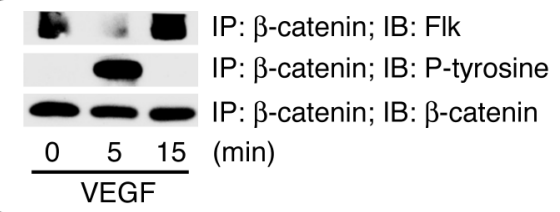

C

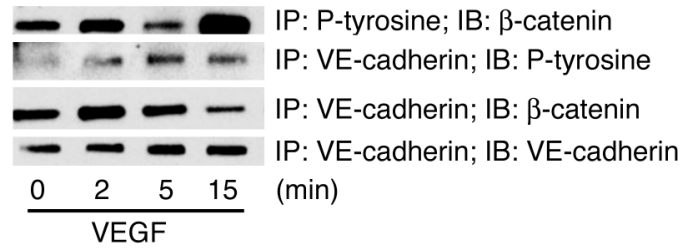

D

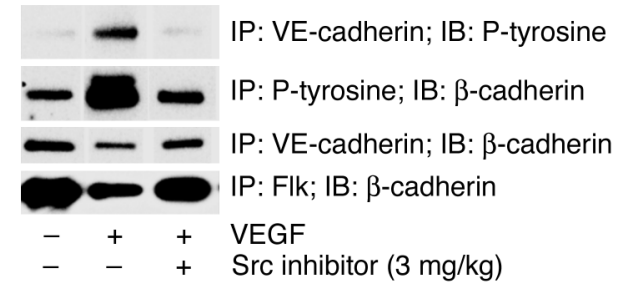

E

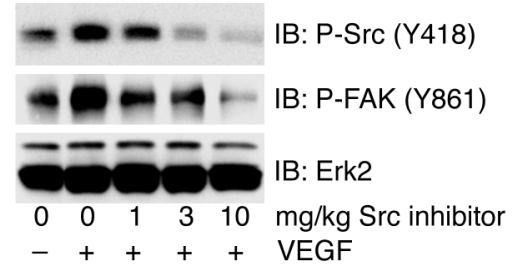

Figure 7

Biochemical signaling in vivo following VEGF is rapid and transient. (A-C) Immunoprecipitation (IP) and immunoblotting (IB) reveal a preformed Flk-cadherin-catenin complex that becomes phosphorylated and dissociates upon systemic VEGF stimulation in mice. (D) Src is required for these VEGF-mediated signaling events, as the Flk-cadherin-catenin complex remains intact in mice pretreated with the Src inhibitor PP1 before VEGF injection. (E) PP1 blocks VEGF-induced Src activity in a dose-dependent way, as assessed by phosphorylation of Src on Y418 and the Src substrate FAK on Y861. Each time point in this figure represents an individual mouse injected with VEGF and sacrificed after a particular duration, although the data shown are representative of at least three separate experiments. Because the events we measure appear to be rapid and transient, we do observe some variation between individual experiments, but we consistently measure phosphorylation events within 2-5 minutes that decrease after 15-30 minutes.

findings that Src inhibition provides protection in terms of edema and infarct size following MI (Figure 1C).

Following MI, VEGF expression likely continues for several hours. To more closely mirror this prolonged exposure to VEGF, healthy mice were injected with VEGF every 30 minutes for 2 hours and were evaluated for the presence of the Flk-cadherin complex and endothelial gaps. While a single VEGF injection produced a reversible, rapid, and transient signaling response that returned to baseline by 15 minutes (Figure 7A), four VEGF injections (one every 30 minutes) produced a prolonged signaling response that was maintained for at least 30 minutes. Specifically, Erk phosphorylation and dissociation of Flk-catenin persisted for 30 minutes following the final injection of VEGF (Figure 8A). At the ultrastructural level, this treatment created damage similar to that observed 24 hours after MI. In this case, we found platelet adhesion, neutrophils, and significant myocyte damage, as well as numerous electron-lucent ECs, many of which were swollen to occlude the vessel lumen (Figure 8, B and C). Taken together, our results indicate that a single injection of VEGF is sufficient to induce, 30 minutes later, an ultrastructure similar to that observed 3 hours after MI. However, longer VEGF exposure (four injections over 2 hours) elicited vascular remodeling similar to that seen in tissues 24 hours after MI.

\section{Proposed role of Src inbibition in the sequence of events following acute $M I$}

We have assembled a schematic diagram that shows our proposed sequence of events following acute MI. The role that SFKs play in regulating VE-cadherin and endothelial gaps and their short- and long-term effects on cardiac tissue are outlined in Figure 9.

\section{Discussion}

VEGF promotes both VP and the growth of new blood vessels. However, prior to promoting revascularization, VEGF has a profound and immediate effect on ischemic tissues by including a VP response that can lead to edema. We contend that this primary VP response and the associated edema can be detrimental to the ischemic tissue. Supporting this notion is the finding that mice deficient in pp60Src, while showing a normal angiogenic response to VEGF, show no VEGF-mediated VP response or edema and consequently have minimal infarcts following ischemic stroke (3) or MI (shown here). In this report, we have presented both ultrastructural and biochemical data supporting a role for Src kinase in the biochemical regulation of EC barrier function, a process we have shown here is directly relevant to the extent of injury following MI.

While our findings indicate that VEGF-mediated Src signaling contributes to disease progression following MI, recent studies suggest that VEGF gene therapy is beneficial to ischemic tissue in animals and man (26). At first glance these findings appear at odds, yet both conclusions may be valid, although on different time scales following initial injury. For example, VEGF gene therapy was initiated days following ischemic injury in previously damaged tissues in which vessel collateralization proved to be beneficial $(26,27)$. In contrast, the window for Src inhibition to block VEGF-mediated VP appears to be relatively early following injury (within 3-6 hours following MI). In this case, the goal is to limit the extent of the initial injury, whereas VEGF-mediated gene therapy is designed to revascularize previously damaged tissue. 
A

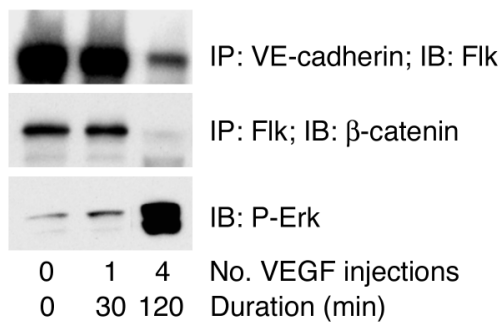

B

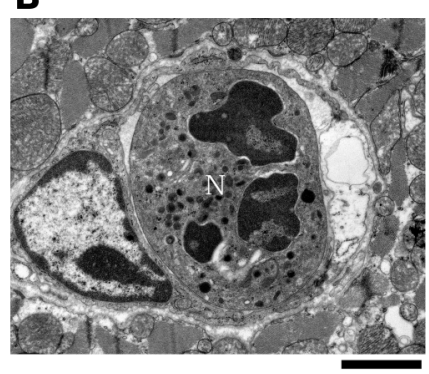

\section{C}

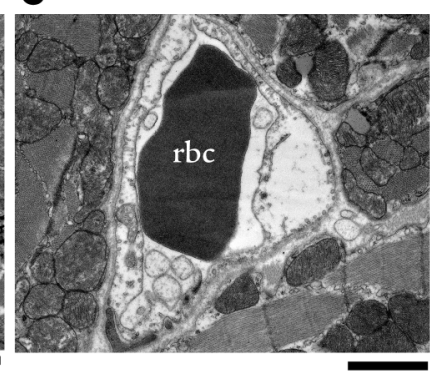

VEGF expression in vivo. VEGF is expressed in vivo in response to a variety of factors (cytokines, oncogenes, and hypoxia) and acts to induce permeability and angiogenesis as well as EC proliferation, migration, and protection from apoptosis (28). Tumors produce large amounts of VEGF which can be detected in the blood stream (29). In fact, blood vessels within or near tumors share many of the features seen in our studies following VEGF injection, such as fenestrated endothelium, open interendothelial junctions, and clustered fused caveolae (30). Serum levels of VEGF in patients with various cancers can range from 100 to $3,000 \mathrm{pg} / \mathrm{ml}$ (29), while local cell or tissue VEGF levels can be 10-100 times higher (31). In patients after an MI, serum VEGF levels have been reported between 100 and $400 \mathrm{pg} / \mathrm{ml}$ and are higher in patients with acute MI versus stable angina (32). As for some primary and metastatic tumors (33), local VEGF levels in the peri-infarct region may well exceed serum levels. The VEGF dose we administered $(167 \mu \mathrm{g} / \mathrm{kg}$, or approximately 2,500 $\mathrm{pg} / \mathrm{ml}$ for an adult mouse) may be equivalent to that experienced in local regions of increased VEGF expression. In fact, the numerous ultrastructural similarities observed between VEGF injection and MI

\section{Figure 9}

Sequence of cellular and molecular events following MI. The link between edema and poor clinical outcome following myocardial infarction has remained poorly understood. We propose a pathway in which hypoxiadriven VEGF expression following MI leads to edema and cardiac damage. Upon VEGF stimulation, Src activity is required for the disruption of a preformed Flk-cadherin-catenin complex, which loosens EC-cell contacts and permits extravasation of serum and blood cells. The resulting edema and inflammation increase interstitial pressure, which reduces local blood flow and causes further hypoxia. Endothelial gaps also expose basal lamina, attracting platelets that adhere, become activated, and could release VEGF locally. Platelet aggregates form microthrombi that can limit blood flow through smaller vessels, thereby increasing hypoxia and contributing to the expansion of cardiac damage and infarcted tissue that is associated with poor clinical outcome. Src blockade delivered within the first few hours following vessel occlusion could prevent VEGF-induced dissociation of the Flk-cadherin-catenin complex and preserve endothelial barrier integrity, thereby eliminating the further damage and infarct expansion beyond the initial ischemic boundary.

\section{Figure 8}

Multiple VEGF injections produce a persistent permeability response. (A) Signaling events following a single VEGF injection are transient and return to baseline by 30 minutes, while prolonged VEGF exposure over 2 hours yields a lasting signaling response. The Flk-cadherin and Flkcatenin complexes remain dissociated after prolonged VEGF exposure, and Erk phosphorylation is sustained. (B) Repeated VEGF injections over 2 hours elicited vascular remodeling similar to that seen in cardiac tissue 24 hours following MI. Microvessel with neutrophil and swollen electron-lucent EC (right side). (C) Vessel with swollen ECs partially occluding lumen. Scale bars: $2 \mu \mathrm{m}$.

support this claim. In fact, our data may explain the findings that some cancer patients have increased thrombotic disease, as increased VEGF accumulation in the circulation would instigate a VP response that attracts platelets and leads to loss of blood flow. In addition, our findings may account for the pleural effusion and general edema associated with late-stage cancer. Thus, blocking Src may have a profound effect on cancer-related edematous disease.

Sequence of events following MI or VEGF injection. Our findings are consistent with the notion that an early blockade of SFKs following MI can have both short- and long-term benefit to cardiac tissue (Figure 9). We show that the early events following MI initiate a biochemical/biological cascade that results in accumulation of edema and tissue damage, followed by fibrosis and remodeling of the heart tissue (Figure 9). In some cases, this injury leads to mortality. By limiting the VP component of the injury early on, one might expect less remodeling of the cardiac tissue leading to a minimal level of fibrosis. The fact that blockade of a single coronary vessel typically leads to a gradual growth of the

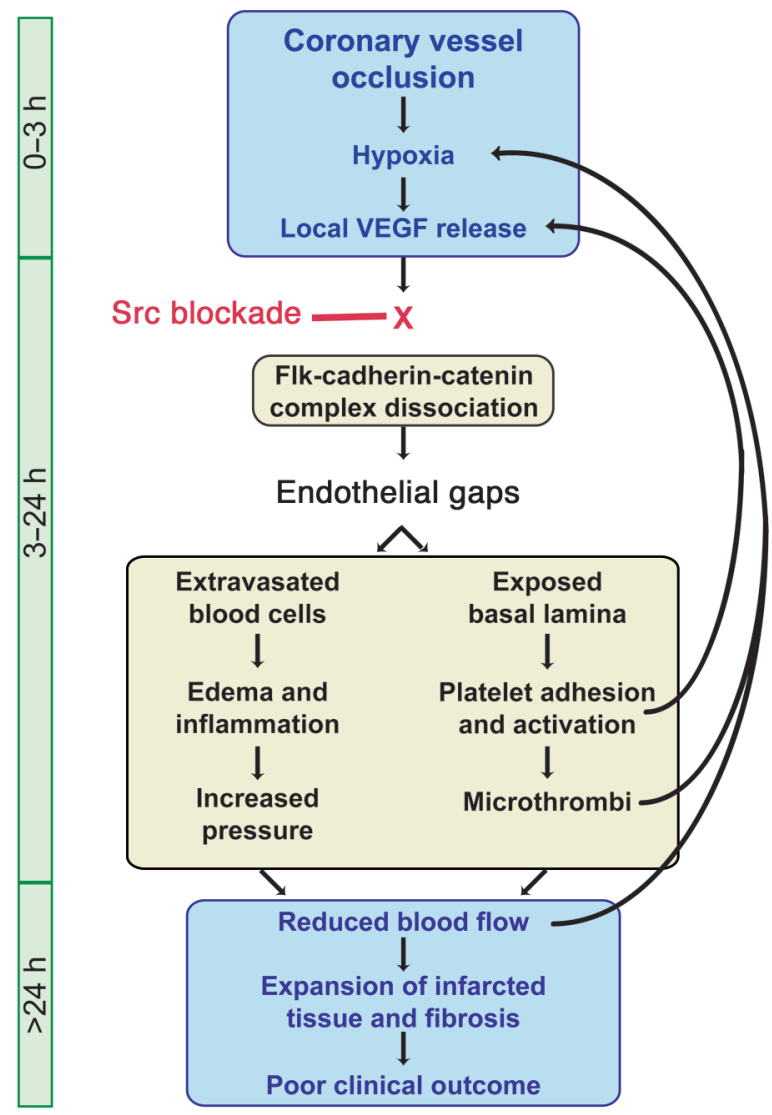


infarct zone, fibrosis, and in some cases death, an early intervention to block VP/edema may provide long term protection and benefit.

In an attempt to identify whether VEGF could account for some of the EC ultrastructure seen following MI, healthy animals were injected systemically with VEGF. In fact, a VEGF injection produced many of the ultrastructural effects observed in cardiac blood vessels following MI. Src blockade not only suppressed these events following MI but also did so after systemic VEGF injection. Other contributors to VEGF-induced VP may include caveolae or vesiculo-vacuolar organelles (34) and fenestrations (35). In addition, inhibition of actin/myosin ATPase activity would block the contractile force necessary to produce EC VP. Src kinase activity could also contribute directly or indirectly to these mechanisms of VP.

Effect of VEGF on blood vessel ultrastructure. Our study is the first to our knowledge to show the ultrastructural changes in EC lining small blood vessels in the heart following MI or systemic injection of VEGF. These observations are consistent with previous reports evaluating the ultrastructure of skin (35), muscle (35), and brain (36) from VEGFtreated animals. Our model of a single injection of VEGF was helpful in dissecting the initial blood vessel response to VEGF, while the multiple-injection model mirrored the effects of longer VEGF exposure on the vascular compartment and therefore may better recapitulate the events associated with VEGF production following MI.

Ultrastructural description of myocyte injury following MI has been reported previously. There is interdigitation of perfused and ischemic tissue in the ischemic border, rather than a smooth delineation radiating outward from the infarct zone (37). Myocyte mitochondria are damaged following MI (38), with deterioration of the cristae similar to that described here. The consequences of VP are observed by examining the injury response 24 hours after MI, when myocyte and blood vessel damage is more prevalent than the initial endothelial barrier dysfunction observed by 3 hours. In the periinfarct zone where VEGF is overexpressed, we have shown that Src inhibition reduces tissue edema and permeability. VEGF administration alone is sufficient to provoke such damage, whether by a onetime injection or by prolonged expression from multiple injections.

The molecular basis of Src in VEGF-mediated VP. A complex between VEcadherin and Flk (KDR/VEGFR-2) has been observed under specific cell culture conditions in vitro $(25,39,40)$. We have confirmed the presence of such a complex in vivo in unstimulated blood vessels. However, we have shown here that this molecular complex immediately dissociates following VEGF stimulation, an event that depends on Src kinase activity. Src in its active form is known to be recruited to Flk upon binding of VEGF (8). Therefore, it is conceivable that active Src associated with Flk may account for the tyrosine phosphorylation of VE-cadherin and $\beta$-catenin, leading to dissociation of the junctional complex. Supporting this notion, previous studies have indicated that such phosphorylation can dissociate a cadherin junctional complex (21). While systemic VEGF administration allows us to evaluate the dissociation of a Flk/cadherin complex within 2 minutes following VEGF injection, the pathological events following MI make this complex difficult to isolate or identify. For example, VEGF levels likely take 2-4 hours to appear after the injury and gradually accumulate over time. Thus, it is likely that the integrity of the vascular compartment is in constant flux in the hours following MI, making it difficult to isolate or monitor a transient complex between Flk and VE-cadherin.

Therapentic implications. Like Src inhibitors, VEGF receptor antagonists may be expected to reduce ischemia-related VP (41). While Src inhibitors may affect a greater number of cell types, including those that do not have VEGF receptors, we contend that blocking Src affects only one of several signaling pathways downstream of VEGF receptor activation. In cultured ECs, nanomolar concentrations of SKI-606 can selectively block VEGF-induced Src and FAK phosphorylation events without disrupting Flk or MAPK activation (data not shown). This supports our previous finding that Src may regulate VEGF-induced VP without necessarily influencing neovascularization. This may explain why Src-deficient animals show normal angiogenesis but no VP response to VEGF (4).

Involvement of other SFK members. Src, Fyn, and Yes are ubiquitously expressed, while the remaining SFK members are expressed mainly in hemopoietic cells. We have previously shown that mice lacking Src or Yes (but not Fyn) lack VEGF-mediated VP (4). Accordingly, mice lacking Src (but not Fyn) are protected following ischemic stroke (3). Therefore, aside from $\mathrm{Src}$, the most likely member involved in the protection to cardiac blood vessels and myocytes offered by pharmacological SFK inhibition is Yes. However, blockade of SFK activity in hemopoietic cells may also play a role. SFK members Fyn, Lyn, Src, Yes, and Hck are expressed in platelets, and recent literature suggests blockade of their activity may influence platelet-platelet (42), platelet-neutrophil (43), or platelet-leukocyte (44) adhesion events. This area of research warrants further investigation, as pharmacological inhibitors that selectively block individual SFK members may represent a novel therapeutic approach for ischemic disease as well as other pathologies.

Conclusions. Disruption of Src appeared to prevent the VEGF-mediated disassembly of a biochemical complex between VE-cadherin and Flk. By maintaining this complex in the presence of VEGF, Src inhibitors appear to limit VP, edema, and long-term damage to cardiac tissue. Together, these findings suggest that Src-mediated, VEGF-induced VP represents a novel therapeutic target, addressing a heretofore poorly understood and lethal consequence of acute MI.

\section{Acknowledgments}

We thank Ricardo Fausto, Jianhua Cui, Marta Bosch-Marce, Chris Jacob, Jann La Vorgna, Theresa Fassel, and The Core Microscopy Facility at TSRI for technical assistance. The authors wish to recognize the original contribution by the late Jeffrey Isner, whose early inspiration and collaboration prompted these experiments. Although Jeffrey is not able to see the results of this work progress to publication, the ongoing projects serve in part as his legacy and remind all involved of his great impact on the field of cardiovascular research. This is manuscript number 15745-IMM from The Scripps Research Institute. S. Weis was supported by NIH fellowship 1F32HL6970102. S. Shintani was supported by the Banyu Fellowship Award in Cardiovascular Medicine sponsored by Banyu Pharmaceutical Co. and The Merck Company Foundation. D. Burstein and N. Himes were supported by NIH grant HL63609. The MRI site is supported by NIH grant RR14792. D. Losordo was supported by NIH grants HL-53354, HL-57516, HL-60911, HL-63414, HL-63695, AG-16332, and HL66957. D. Cheresh was supported by NIH grants CA45726, CA95262, P01-EY14174, P01-CA78045, R37-CA50286, and P01-HL57900.

Received for publication December 2, 2003, and accepted in revised form January 6, 2004.

Address correspondence to: David Cheresh, Department of Immunology, The Scripps Research Institute, 10550 N. Torrey Pines Road, La Jolla, California 92037, USA. Phone: (858) 784-8281; Fax: (858) 784-8926; E-mail: cheresh@scripps.edu.

Sara Weis and Satoshi Shintani contributed equally to this work. 
1. Garcia-Dorado, D., and Oliveras, J. 1993. Myocardial oedema: a preventable cause of reperfusion injury? Cardiovasc. Res. 27:1555-1563.

2. Senger, D.R., Perruzzi, C.A., Feder, J., and Dvorak, H.F. 1986. A highly conserved vascular permeability factor secreted by a variety of human and rodent tumor cell lines. Cancer. Res. 46:5629-5632.

3. Paul, R., et al. 2001. Src deficiency or blockade of Src activity in mice provides cerebral protection following stroke. Nat. Med. 7:222-227.

4. Eliceiri, B.P., et al. 1999. Selective requirement for Src kinases during VEGF-induced angiogenesis and vascular permeability. Mol. Cell. 4:915-924.

5. Breviario, F., et al. 1995. Functional properties of human vascular endothelial cadherin (7B4/cadherin$5)$, an endothelium-specific cadherin. Arterioscler. Thromb. Vasc. Biol. 15:1229-1239.

6. Behrens, J., et al. 1993. Loss of epithelial differentiation and gain of invasiveness correlates with tyrosine phosphorylation of the E-cadherin/beta-catenin complex in cells transformed with a temperaturesensitive v-SRC gene. J. Cell Biol. 120:757-766.

7. Hamaguchi, M., et al. 1993. p60v-src causes tyrosine phosphorylation and inactivation of the $\mathrm{N}$-cadherincatenin cell adhesion system. EMBO J. 12:307-314.

8. Chou, M.T., Wang, J., and Fujita, D.J. 2002. Src kinase becomes preferentially associated with the VEGFR, KDR/Flk-1, following VEGF stimulation of vascular endothelial cells. BMC Biochem. 3:32

9. Banks, R.E., et al. 1998. Release of the angiogenic cytokine vascular endothelial growth factor (VEGF) from platelets: significance for VEGF measurements and cancer biology. Br. J. Cancer. 77:956-964.

10. Hanke, J.H., et al. 1996. Discovery of a novel, potent, and Src family-selective tyrosine kinase inhibitor. Study of Lck- and FynT-dependent T cell activation. J. Biol. Chem. 271:695-701.

11. Golas, J.M., et al. 2003. SKI-606, a 4-anilino-3-quinolinecarbonitrile dual inhibitor of Src and Abl kinases, is a potent antiproliferative agent against chronic myelogenous leukemia cells in culture and causes regression of K562 xenografts in nude mice. Cancer Res. 63:375-381.

12. Boschelli, D.H., et al. 2001. Optimization of 4-phenylamino-3-quinolinecarbonitriles as potent inhibitors of Src kinase activity. J. Med. Chem. 44:3965-3977.

13. Kawamoto, A. et al. 2003. Intramyocardial transplantation of autologous endothelial progenitor cells for therapeutic neovascularization of myocardial ischemia. Circulation. 107:461-468.

14. Soriano, P., Montgomery, C., Geske, R., and Bradley, A. 1991. Targeted disruption of the c-src proto-oncogene leads to osteopetrosis in mice. Cell. 64:693-702.

15. Fishbein, M.C., et al. 1981. Early phase acute myocardial infarct size quantification: validation of the triphenyl tetrazolium chloride tissue enzyme staining technique. Am. Heart J. 101:593-600.

16. Albers, J., et al. 2001. 3D evaluation of myocardial edema: experimental study on 22 pigs using magnet- ic resonance and tissue analysis. Thorac. Cardiovasc. Surg. 49:199-203.

17. Schiller, N.B., et al. 1989. Recommendations for quantitation of the left ventricle by two-dimensional echocardiography. American Society of Echocardiography Committee on Standards, Subcommittee on Quantitation of Two-Dimensional Echocardiograms. J. Am. Soc. Echocardiogr. 2:358-367.

18. Wendland, M.F., Saeed, M., Lund, G., and Higgins, C.B. 1999. Contrast-enhanced MRI for quantification of myocardial viability. J. Magn. Reson. Imaging. 10:694-702.

19. Gould, K.E., et al. 2002. Heart failure and greater infarct expansion in middle-aged mice: a relevant model for postinfarction failure. Am. J. Physiol. Heart Circ. Physiol. 282:H615-H621.

20. Webb, N.J., Myers, C.R., Watson, C.J., Bottomley, M.J., and Brenchley, P.E. 1998. Activated human neutrophils express vascular endothelial growth factor (VEGF). Cytokine. 10:254-257.

21. Esser, S., Lampugnani, M.G., Corada, M., Dejana, E., and Risau, W. 1998. Vascular endothelial growth factor induces VE-cadherin tyrosine phosphorylation in endothelial cells. J. Cell Sci. 111:1853-1865.

22. Al Moustafa, A.E., Yen, L., Benlimame, N., and Alaoui-Jamali, M.A. 2002. Regulation of E-cadherin/catenin complex patterns by epidermal growth factor receptor modulation in human lung cancer cells. Lung Cancer. 37:49-56.

23. Gamble, J.R., et al. 2000. Angiopoietin-1 is an antipermeability and anti-inflammatory agent in vitro and targets cell junctions. Circ. Res. 87:603-607.

24. Debiais, F., et al. 2001. Fibroblast growth factor-2 (FGF-2) increases $\mathrm{N}$-cadherin expression through protein kinase $\mathrm{C}$ and Src-kinase pathways in human calvaria osteoblasts. J. Cell. Biochem. 81:68-81.

25. Shay-Salit, A., et al. 2002. VEGF receptor 2 and the adherens junction as a mechanical transducer in vascular endothelial cells. Proc. Natl. Acad. Sci. U. S. A 99:9462-9467.

26. Vale, P.R., Isner, J.M., and Rosenfield, K. 2001. Therapeutic angiogenesis in critical limb and myocardial ischemia. J. Interv. Cardiol. 14:511-528.

27. Losordo, D.W., et al. 2002. Phase $1 / 2$ placebo-controlled, double-blind, dose-escalating trial of myocardial vascular endothelial growth factor 2 gene transfer by catheter delivery in patients with chronic myocardial ischemia. Circulation. 105:2012-2018.

28. Neufeld, G., Cohen, T., Gengrinovitch, S., and Poltorak, Z. 1999. Vascular endothelial growth factor (VEGF) and its receptors. FASEB J. 13:9-22.

29. Dunst, J., et al. 1999. Low hemoglobin is associated with increased serum levels of vascular endothelial growth factor (VEGF) in cancer patients. Does anemia stimulate angiogenesis? Strablenther. Onkol. 175:93-96.

30. Roberts, W.G., and Palade, G.E. 1997. Neovasculature induced by vascular endothelial growth factor is fenestrated. Cancer Res. 57:765-772.
31. Cheng, W.F., et al. 1999. Vascular endothelial growth factor in cervical carcinoma. Obstet. Gynecol. 93:761-765.

32. Shintani, S., et al. 2001. Mobilization of endothelial progenitor cells in patients with acute myocardial infarction. Circulation. 103:2776-2779.

33. Stockhammer, G., et al. 2000. Vascular endothelial growth factor (VEGF) is elevated in brain tumor cysts and correlates with tumor progression. Acta Neuropathol. (Berl.) 100:101-105.

34. Feng, D., Nagy, J.A., Hipp, J., Dvorak, H.F., and Dvorak, A.M. 1996. Vesiculo-vacuolar organelles and the regulation of venule permeability to macromolecules by vascular permeability factor, histamine, and serotonin. J. Exp. Med. 183:1981-1986.

35. Roberts, W.G., and Palade, G.E. 1995. Increased microvascular permeability and endothelial fenestration induced by vascular endothelial growth factor. J. Cell. Sci. 108:2369-2379.

36. Dobrogowska, D.H., Lossinsky, A.S., Tarnawski, M., and Vorbrodt, A.W. 1998. Increased blood-brain barrier permeability and endothelial abnormalities induced by vascular endothelial growth factor. J. Neurocytol. 27:163-173.

37. Axford-Gatley, R.A., and Wilson, G.J. 1988. The "border zone" in myocardial infarction. An ultrastruc tural study in the dog using an electron-dense blood flow marker. Am. J. Pathol. 131:452-464.

38. Weitbrecht, M., Schaper, J., Zanker, K., Blumel, G., and Mathes, P. 1983. Morphology and mitochondrial function of the surviving myocardium following myocardial infarction in the cat. Basic Res. Cardiol. 78:423-434.

39. Carmeliet, P., et al. 1999. Targeted deficiency or cytosolic truncation of the VE-cadherin gene in mice impairs VEGF-mediated endothelial survival and angiogenesis. Cell. 98:147-157.

40. Zanetti, A., et al. 2002. Vascular endothelial growth factor induces SHC association with vascula endothelial cadherin: a potential feedback mechanism to control vascular endothelial growth factor receptor-2 signaling. Arterioscler. Thromb. Vasc. Biol. 22:617-622.

41. van Bruggen, N., et al. 1999. VEGF antagonism reduces edema formation and tissue damage afte ischemia/reperfusion injury in the mouse brain J. Clin. Invest. 104:1613-1620.

42. Obergfell, A., et al. 2002. Coordinate interactions of Csk, Src, and Syk kinases with $\alpha \operatorname{IIb} \beta 3$ initiate integrin signaling to the cytoskeleton. J. Cell Biol. 157:265-275

43. Evangelista, V., Manarini, S., Coller, B.S., and Smyth, S.S. 2003. Role of P-selectin, $\beta 2$-integrins, and Src tyrosine kinases in mouse neutrophil-platelet adhesion. J. Thromb. Haemost. 1:1048-1054.

44. Piccardoni, P., et al. 2001. Platelet/polymorphonuclear leukocyte adhesion: a new role for SRC kinases in Mac-1 adhesive function triggered by P-selectin. Blood. 98:108-116. 